\title{
Trajectories of functional disability for the elderly in Britain
}

\author{
Robert French \\ R.French@bris.ac.uk
}

Fiona Steele

\author{
Centre for Multilevel Modelling, University of Bristol
}

Department of Statistics, London School of Economics and Political Science

\begin{abstract}
This study uses an innovative approach to characterise trajectories of functional disability over the final stages of the life course. We use data from the British Household Panel Survey (BHPS), an annual household survey of all adults in a representative sample of British households from 1991-2008. The analysis focuses on the sub-sample of elderly household members who were aged from 65 to 74 in any of the 18 waves of data, with a final sample of 3,671 individuals contributing a total of 13,982 person years. As in previous research, we estimate latent growth curves, but extend the standard model to incorporate a measurement model for the latent outcome variable 'functional disability'. We identify accelerating trajectories of functional disability for a representative sample of elderly individuals separately by gender. We show that sociooccupational classification is associated with the level of initial functional disability and to a lesser extent the increase in functional disability with age. The contribution of this paper is to explore the use of a measurement model to exploit the variation between items in discriminatory power for identifying an individual's functional disability. Further we are able to explicitly test for temporal measurement invariance in functional disability i.e. to what extent the items consistently measure the latent variable as people age.
\end{abstract}

Keywords: Ageing, activities of daily living, health trajectories, Britain, British Household Panel Survey (BHPS), structural equation model (SEM), growth model, measurement model, temporal measurement invariance

\section{Introduction}

The ageing population in the UK is the result of a reduced birth rate and delayed mortality. Delayed mortality may result in a change in the prevalence of morbidity, either increasing (Verbrugge, 1984) or decreasing (Fries, 1980), which has implications for health care costs. For a well-informed policy response to this ageing population one needs welldefined measures of health for the elderly, and to establish how these measures progress with age, and how the level and nature of change with age differs between individuals.

The ageing process is typically represented by a trajectory of declining health, defined by increasing disability (Grundy \& Glaser, 2000), or diminishing quality of life (Zaninotto, Falaschetti, \& Sacker,
2009), self-rated health (Sacker, Worts, \& McDonough, 2011), physical performance (Payette et al., 2011), or ability to carry out everyday activities (Haas, 2008). In this study we are concerned with a functional definition of health how far health limits an individual's ability to enjoy a normal life - rather than a medical definition or diagnosis, since it allows comparability between individuals across a variety of different health conditions (Burchardt, 2000). This is typically measured using questions regarding individuals' ability to undertake everyday tasks over several domains. The first core set of such questions is the activities of daily living (ADL) (Katz, Ford, Moskowitz, Jackson, \& Jaffe, 1963), though the ADL term is now used generically to describe a wide 
variety of question sets that attempt to capture the same construct. Extensions to ADL include the instrumental activities of daily living (iADL) (Lawton \& Brody, 1969) which includes higher level tasks and SF-36 (Ware \& Sherbourne, 1992) which captures social functioning. Because ADL are measured over different domains it is useful to combine these items into a single metric of functional disability for analysing changes in health.

We argue that the methods used to combine the ADL scores for models of change in functional disability have two important limitations. Firstly, these studies use simple aggregations of individual items, such as the sum of ADL scores, to create the single metric for analysing change, typically assigning equal or arbitrarily-chosen differential weights to each activity. This approach ignores variability between items in their relative difficulty and in their ability to discriminate between individuals with different levels of physical functioning. Secondly, previous studies have assumed that the difficulty and discriminatory power of items is the same at each age, widely referred to as temporal/longitudinal measurement invariance. Departures from this assumption due to changes in the relationship between the observed items and the underlying construct with age observed in cross-sectional studies (LaPlante, 2010) will confound attempts to identify growth patterns.

We propose a measurement model to more fully capture the underlying structure of functional disability. The measurement model includes parameters representing the difficulty and discriminatory power of each item. Making explicit the relationship between the items and the latent construct (physical functioning) in this way also allows exploration and testing of temporal measurement invariance. Provided that temporal measurement invariance holds, the measurement model can then be combined with a growth model for latent physical functioning. We allow our models of change in functional disability to differ by gender and socioeconomic status. We estimate separate models for each gender because we expect functional disability trajectories for women to show worse health for biological, psychological and sociological factors (Nathanson, 1975). We allow trajectories to vary by socioeconomic status as we expect a social gradient through accrued exposure to risk factors, both in terms of direct effects from certain types of employment, but also from the indirect risk factors and mediating factors associated with class (Nilsson, Avlund, \& Lund, 2010).

\section{Review of approaches to modelling trajectories of physical functioning}

Trajectories of functional disability can be estimated using either a multilevel model (MLM) or structural equation model (SEM). In their simplest form, these models are equivalent (Curran, 2003; Steele, 2008). Both allow for individual-specific trajectories with normally distributed latent variables representing individual departures from the intercept and slope of an overall growth curve, and both can be extended to allow for nonlinear growth. These latent variables are usually referred to as random effects in MLM and factors in SEM.

In a MLM for growth the repeated health measurements are viewed as a two-level hierarchical structure with occasions nested within individuals and age is treated as a time-varying explanatory variable (Goldstein \& Woodhouse, 2001). The advantage of the MLM approach is that it is very flexible, with possible extensions to the basic growth curve including allowance for additional levels of clustering and betweenindividual variation in the timing of measurements at a given occasion. Individuals not present at all measurement points can be included under a 'missing at random' assumption (Little \& Rubin, 2002), however those with missing items within a wave require multiple imputation of missing values in order for that wave to be included.

In a SEM for growth, the measures at each occasion are treated as the observed indicators of the unobserved latent growth factors, i.e. latent variables for the individual-specific intercepts and slopes. The advantage of the SEM approach is the ability to include additional latent variables, for example to allow for measurement error in outcomes or covariates. It is also straightforward using SEM software to incorporate individuals with incomplete data within waves.

Several studies have fitted a latent growth curve model to trajectories of functional disability. Li (2005) estimates a two-level random effects model of 'ADL disability' using the Michigan's Medicaid Waiver Program of individuals aged 65+ measured every few months from 1999 to 2003. They find evidence of an accelerating trajectory of ADL disability for the whole sample. Park et al. (2008) use a similar model of 'functional status' for the 
University of Alabama at Birmingham Ageing Study which surveys individuals aged 65+ every 6 months from 1999 to 2004. They also find increasing and accelerating functional disability. Mendes de Leon et al. (2002) estimate a MLM of 'ADL disability' using the Women's Health and Ageing Study which follows women aged $65+$ for 24 consecutive weekly assessments in 1992. They find a linear increase in functional disability over this much shorter time frame. Haas (2008) estimates a latent growth curve of 'functional limitations' using the Health and Retirement Study which follows individuals aged 6171 at baseline annually from 1992-2002, and find an increasing and accelerating trajectory for functional limitations.

These papers use unconditional models as a baseline and to identify functional form, and conditional models to quantify how these trajectories differ by individual characteristics. Stuck et al. (1999) review the individual risk factors for ADL decline. In this study we focus on two of the most common: gender and SES. Although females live longer than males, women generally have higher reported illness (Nathanson, 1975). There are many reasons for these differences, for example biological factors such as genes and hormones make males more susceptible to diseases that result in death, e.g. heart disease, while women are more likely to suffer from conditions which impact on reported health but not death, e.g. arthritis (Case \& Paxson, 2005). Moreover, there are gender differences in acquired risks: for example men are more likely to smoke and drink while females are more likely to be overweight and face stress (Verbrugge, 1989). The SES gradient in health arises from direct risk factors associated with occupation, e.g. physical hazards and psychosocial stressors at work, but also from risk behaviours associated with class, e.g. smoking and heavy alcoholic drinking (Feng et al., 2013). Over the life course we expect the SES effect to increase as exposure lengthens (Sacker, Clarke, Wiggins, \& Bartley, 2005), but once an individual retires the SES effects accrued during the working life may diminish as exposure to certain risk factors associated with work cease (House, Kessler, \& Herzog, 1990).

\section{Measurement of physical functioning in longitudinal studies}

All of the methods of studying longitudinal change in functional disability discussed above use a single health outcome variable created using answers to a series of questions. The simplest approach to creating a single measure from these multiple questions is to sum ADL scores on each question. For example, Li (2005) uses questions on eight activities, with responses coded between zero (no limitation) and four (maximum limitation). These scores are summed across the eight items to generate the functional disability outcome measure. Using the total ADL score is problematic since each component is given equal weight, thus ignoring variation in the discriminatory power of the different items. Others studies use ad hoc methods to assign different weights to the items. For example, Holstein et al. (2006) measure levels of difficulty for $12 \mathrm{ADL}$ items, and use these to create four categories of functional disability: (i) individuals who can manage all items without difficulty, (ii) individuals who can manage every activity but some with difficulty, (iii) those who need help in at least one category, and (iv) those who need help with two or more activities. Such an approach compounds the problem of equal weighting of different items by then using arbitrary thresholds for categorisation; it also ignores much of the information contained in the responses. We propose to use a measurement model to generate a single metric for functional limitations, which has the advantage that it allows each of the activities to have its own relationship with the latent outcome variable, rather than imposing equal or arbitrary weights.

\section{Methods}

\section{Data and measures}

Data for the study are from the British Household Panel Survey (BHPS), an annual household survey of all adults in a representative sample of British households from 1991-2008 (Institute for Social and Economic Research, 2010). Elderly household members (aged 65 or over) were asked additional questions on their ability to carry out activities of daily living and these formed the sample for analysis. We have 1,712 males and 1,959 females, contributing a total of 13,982 person years. Individuals not present for all waves of the survey were still included in the analysis, as were cases with missing data on some of the ADL measures for a particular year. Wave non-response and missing data on ADL items are handled using maximum likelihood methods under a missing at 
random assumption (Little \& Rubin, 2002) in Mplus (Muthén \& Muthén, 1998-2012).

Our observed indicators of physical functioning are the ADL items: 'get in and out of bed', 'cut toenails', 'get up and down stairs or steps', 'bath, shower or wash all over', 'get around the house', and 'walk down the road'. The score for each ADL item was constructed from responses to two questions: whether the individual is able to carry out an ADL (Q1 coded unaided, aided or not at all) and, for those who answered "unaided", the level of difficulty in performing the ADL (Q2 coded very easy, fairly easy, fairly difficult or very difficult). Thus for each ADL we can construct a six-point score, ranging from zero for those with the least disability who could carry out the ADL unaided (Q1) and very easily (Q2) to five for those with the most disability who could not carry out the ADL at all (Q1 only).

Although measurements of ADL were available for all individuals aged 65 and above, we only include individuals at ages 65 to 74 . For example, with 18 years of data, an individual aged 65 at wave one may have $A D L$ measurements until they were aged 83 (at wave 18), but we discard data for ages 75 and above. Likewise individuals that entered the survey aged 75 or above were not included in our analysis. By restricting analysis to smaller, more homogenous age-groups we are more likely to satisfy the measurement invariance assumption discussed in the following section - that the measurement model is consistent as individuals age. For the same reason we also estimate separate models for male and females, thus avoiding the assumption that the measurement model has the same form for both genders.

We allow the level and rate of change of the trajectories to differ by SES. The measure of SES used is the National Statistics Socio-Economic Classification (NS-SEC) (Office for National Statistics, 2010) which categorises each individual's final occupation into eight classes. The 'never worked and long term unemployed' category is excluded because this group is likely to have health issues and hence trajectories that are rather different from the majority of the population. We would expect a social gradient in functional disability using NS-SEC due to accumulation of exposure to risk factors over the working life.

\section{Longitudinal structural equation model (SEM) of physical functioning}

In this paper we use a type of SEM known as a multiple indicator growth model (Chan, 1998; Hancock, Kuo, \& Lawrence, 2001; Wu, Liu, Gadermann, \& Zumbo, 2010). The model consists of two simultaneously estimated components: a measurement model relating responses on the six observed ADL items to a latent variable representing physical functioning, and a growth model for change in the latent variable with age. Separate SEMs were fitted for men and women.

\section{Measurement model}

Let $y_{r t i}$ denote the response on item $r$ at age $t$ for individual $i$. A general longitudinal measurement model can be written

$$
y_{r t i}=\alpha_{r t}+\lambda_{r t} f_{t i}+\epsilon_{r t i}
$$

where $f_{t i}$ is the latent functional disability at age $t$ for individual $i, \alpha_{r t}$ are intercepts, $\lambda_{r t}$ are coefficients or factor loadings, and $\epsilon_{r t i}$ are residuals. The age-specific factors $f_{t i}$ and residuals $\epsilon_{r t i}$ are each assumed to follow multivariate normal distributions. We allow for autocorrelation in both functional disability and individual items across ages. We assume that the covariance between items at a given age $t$ is explained by the common factor $f_{t i}$, so that $\operatorname{cov}\left(\epsilon_{r t i}, \epsilon_{s t i}\right)=0$ for $r \neq s$. To fix the location and scale of $f_{t i}$ we impose the identification constraints $\alpha_{1 t}=0$ and $\lambda_{1 t}=1$.

The model of equation (1), which we refer to as model 1, allows for changes in the underlying structure of functional disability with age through the inclusion of age-specific intercepts and loadings. However, under this model individual trajectories in $f_{t i}$ are difficult to interpret because changes in the true level of physical functioning with age are confounded with changes in its measurement. Before estimating growth trajectories for $f_{t i}$ we therefore test for temporal measurement invariance by considering two increasingly restricted forms of equation (1). In model 2, factor loadings for the same item are constrained to be equal across ages $\left(\lambda_{r t}=\lambda_{r}\right)$. This model assumes metric invariance which can be tested by comparison with the base model 1 . We then consider model 3 with the additional restriction that the intercepts for the same item are fixed across ages $\left(\alpha_{r t}=\alpha_{r}\right)$. A comparison of model 3 and model 2 tests for scalar invariance. The combination of metric and scalar invariance in model 3 is sometimes referred to as strong 
invariance, which is widely considered as an essential prerequisite for examining temporal change in $f_{t i}$.

\section{Testing for temporal measurement invariance}

We test the overall fit of the measurement models using chi-squared $\left(\chi^{2}\right)$ tests, comparing each model with the saturated model which has unconstrained means and covariance matrix. Although the $\chi^{2}$ test is widely used, there are several limitations relevant to our study: (i) the $\chi^{2}$ test statistic is dependent on sample size and sensitive to the size of the correlations between the observed items, with large samples and correlations leading to higher values of $\chi^{2}$, (ii) in a multi-group model (or repeated observation of the same group over time) the $\chi^{2}$ test is sensitive to even minor deviations between the groups' sample covariance matrices, and (iii) the test is based on the assumption that the observed variables have a multivariate normal distribution, with departures from normality leading to higher values of $\chi^{2}$ (Kline, 2005; Vandenberg \& Lance, 2000). These problems with the $\chi^{2}$ test have led to the development of numerous fit indices which are usually considered alongside the $\chi^{2}$ test, many of which are based on the $\chi^{2}$ with adjustments for sample size and model complexity.

For each of these alternative tests of model fit, Vandenberg and Lance (2000) specify the traditional values required to infer good model fit alongside the more stringent thresholds proposed by $\mathrm{Hu}$ and Bentler (1999). We consider both of these thresholds in our analysis. The first of the alternative tests is the Tucker-Lewis index (TLI) (Tucker \& Lewis, 1973) which is less susceptible to sample size and favours parsimonious models. Values of the TLI range between 0 and 1 with higher values indicating better fit, and a traditional threshold of 0.9 or above and a more stringent threshold of 0.95 or above for a good model fit. The second alternative test of fit is the root mean square error of approximation (RMSEA) (Steiger, 1990) which does not require a null model and also adjusts for model complexity. The RMSEA also ranges from 0 to 1 , but with values close to zero indicating a better fit. The traditional threshold value for an acceptable model fit is 0.08 or less, and a more stringent threshold of 0.06 or less. The third alternative test is the standardised root mean square residual (SRMR) (Bentler, 1995) which is sensitive to model specifications among the factor covariances. The SRMR again ranges from 0 to 1 , with lower values indicating better model fit, the traditional threshold for good model fit is 0.10 or less, and a more stringent threshold of 0.08 or less.

In addition to the $\chi^{2}$ test and alternative tests for assessing absolute model fit described above, Vandenberg and Lance (2000) suggest two ways for evaluating relative model fit, in our case the change in model fit arising from adding the temporal measurement invariance constraints of models 2 and 3 . The first test is based on the change in the chi-squared $\left(\Delta \chi^{2}\right)$, where a non-significant difference between models indicates that the additional temporal measurement invariance constraint does not lead to a deterioration in model fit. The second approach is to examine the change in the comparative fit index $(\Delta \mathrm{CFI})$. Cheung and Rensvold (1999) provide guidelines on model fit suggesting that a $\Delta \mathrm{CFI}$ value closer to zero than 0.01 indicates that the more restrictive model is an adequate fit (i.e. the invariance hypothesis should not be rejected), a $\Delta$ CFI of between -0.01 and -0.02 indicates researchers should be suspicious about the invariance assumption, and $\Delta \mathrm{CFI}$ of less than 0.02 suggests that the invariance constraint should be rejected.

\section{Latent growth models with SES effects}

The measurement model shown in equation (1) specifies the relationship between an individual's latent functional disability $f_{t i}$ at age $t$ and their responses on the observed ADL items. Age is centred at the baseline age of 65 . The second part of the SEM (commonly referred to as the 'structural' model) is a growth model for change in this latent variable with age. We consider a nonlinear growth model in which $f_{t i}$ changes as a quadratic function of age and additionally depends on dummy variables for SES $x_{m i}(m=2,3, \ldots, 7)$, taking the first category as the reference. Growth models with a cubic polynomial in age were also considered, but the addition of the cubic term did not lead to a significant improvement in model fit for any of the four samples. We therefore present results for quadratic models. The growth model can be expressed as 


$$
\begin{aligned}
f_{t i}=\beta_{0 i}+\beta_{1 i} t & +\beta_{2 i} t^{2} \\
& +\sum_{m=2}^{7} \gamma_{0 m} x_{m i}+\sum_{m=2}^{7} \gamma_{1 m} x_{m i} t+\sum_{m=2}^{7} \gamma_{2 m} x_{m i} t^{2}+e_{t i}
\end{aligned}
$$

The intercept and coefficients of the quadratic function in age, $\beta_{k i}=\beta_{k}+u_{k i}(k=0,1,2)$, are composed of a fixed part $\beta_{k}$ common to all individuals and an individual-specific random effect $u_{k i}$, where the random effects $\left(u_{0 i}, u_{1 i}, u_{2 i}\right)$ are assumed to follow a trivariate normal distribution. The $e_{t i}$ are independent normally distributed timevarying residuals. The main effects of SES, the coefficients $\gamma_{0 m}$ of $x_{m i}$, allow baseline functional disability (at age 65, $t=0$ ) to depend on SES, while the coefficients of the interactions between SES and $t$ and $t^{2}\left(\gamma_{1 m}\right.$ and $\left.\gamma_{2 m}\right)$ allow the rate of change in functioning with age to vary with SES.

This SEM (Model 4) which combines the measurement model of equation (1) and growth model of equation (2) is the main model of interest. We also estimate a second SEM (Model 5) which constrains the factor loadings to be equal for all items. This is akin to modelling the growth of a functional disability measure which is simply the sum of the scores on each of the items. Thus contrasting Model 4 with Model 5 allows us to see the effect of failure to allow for differences in the discriminatory power of the ADL items when modelling functional disability trajectories.

\section{Results}

Measurement models and evidence for temporal measurement invariance

To test for temporal measurement invariance in our data we estimate three versions of the measurement model with increasingly rigorous constraints. Model 1 is a simple measurement model with no measurement invariance constraints i.e. factor loadings and item intercepts are allowed to vary with age. Absolute model fit statistics for Model 1 are presented in the first panel of Table 1. For both gender groups the $\chi^{2}$ test indicates significant differences between Model 1 and the baseline saturated model (with parameters for the means, variances and covariances for the $6 \mathrm{ADL}$ items measured at 10 time points). The TLI gave weak evidence of good model fit with values below the more stringent threshold for both gender subsamples, with females just above the less stringent while males were below even this threshold. The RMSEA provided the strongest evidence of good model fit, with values for the well below the more stringent threshold for both samples. The SRMSR also provides evidence of good model fit, with values below the more stringent threshold.

Table 1: Tests for temporal measurement invariance

\begin{tabular}{lcc} 
& Males & Females \\
\hline Absolute fit of Model 1: & & \\
Chi-square test statistic, $1395 \mathrm{df}\left(\chi^{2}\right)$ & 4830 & 4317 \\
TLI & 0.892 & 0.910 \\
RMSEA & 0.038 & 0.033 \\
SRMSR & 0.078 & 0.070 \\
Change in model fit between Model 1 and Model 2: & & \\
$\quad$ Chi-square test statistic, $45 \mathrm{df}\left(\Delta \chi^{2}\right)$ & 116 & 132 \\
$\quad$ Change in CFI ( $\Delta$ CFI) & -0.002 & -0.002 \\
Change in model fit between Model 2 and Model 3: & & \\
$\quad$ Chi-square test statistic, $45 \mathrm{df}\left(\Delta \chi^{2}\right)$ & 161 & 169 \\
$\quad$ Change in CFI ( $\Delta$ CFI) & -0.002 & -0.003 \\
\hline$n \quad$ & & 1912
\end{tabular}


Model 2 is a restricted version of model 1 with the factor loadings for each item constrained to be equal for all ages. Figure 1 shows how the estimated factor loadings of model 1 are broadly similar over ages though with a slight upwards trend (which is consistent with all activities becoming more difficult as individuals get older), so it seems reasonable that constraining these to be equal over time may be a sensible assumption. We formally test whether this is assumption holds by comparing the change in model fit between models 1 and 2, in other words whether the differences in the factor loadings of the measurement model by age shown in figure 1 are sufficiently large to make a significant change to model fit. The tests of change in model fit between model 1 and model 2 are shown in the second panel of table 1 . The $\Delta \chi^{2}$ between models 1 and 2 suggests that imposing time invariant factor loadings leads to a significantly worse model fit. However we see only a small $\triangle \mathrm{CFI}$, far below the threshold for metric invariance. Overall we conclude that there is some evidence of metric invariance.

\section{Figure 1: Trajectories of factor loadings $\left(\lambda_{r}\right)$, when allowed to vary by age (Model 1 )}

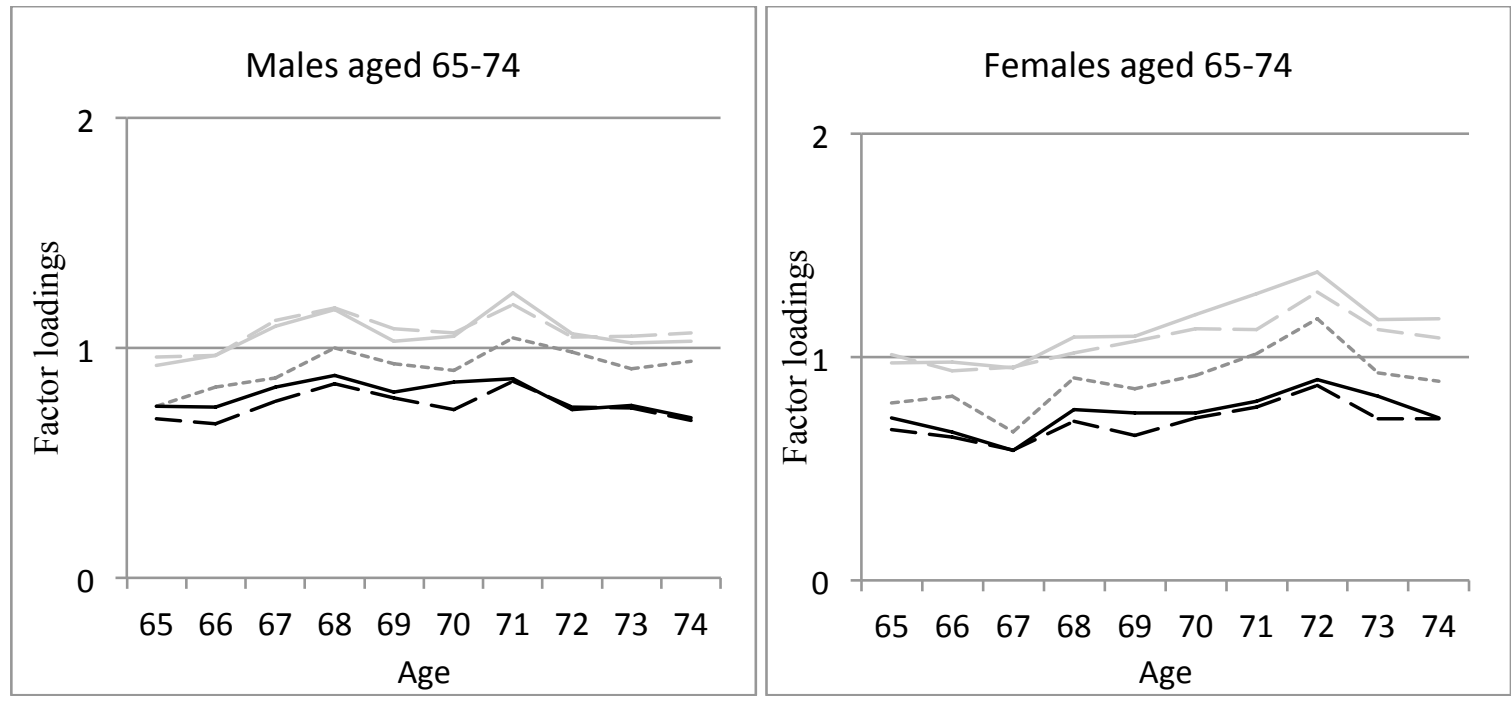

Walk down the road
$-\ldots \quad$ Get up and down stairs
Bathe, shower or wash all over
$-\ldots \quad$ Get around the house
$-\quad$ Get in and out of bed

Model 3 is a more restricted version of model 2 in which the intercepts for each item are constrained to be the same for all ages. The intercepts were allowed to vary by age in models 1 and 2. Figure 2 shows the estimates of the item intercepts by age for model 2 . These show a downward trend in the item intercepts over time. We formally test for scalar invariance - that is whether constraining the item intercepts to be equal over ages is a reasonable assumption - by examining the change in model fit statistics between model 2 and model 3 (see the third panel of table 1). As seen for the contrast between models 1 and 2 the $\Delta \chi^{2}$ indicates a significantly worse model fit, but a very small $\Delta \mathrm{CFI}$ supports the assumption of scalar invariance. 
Figure 2: Item intercepts $\alpha_{r}$, when allowed to vary by age (Model 2)

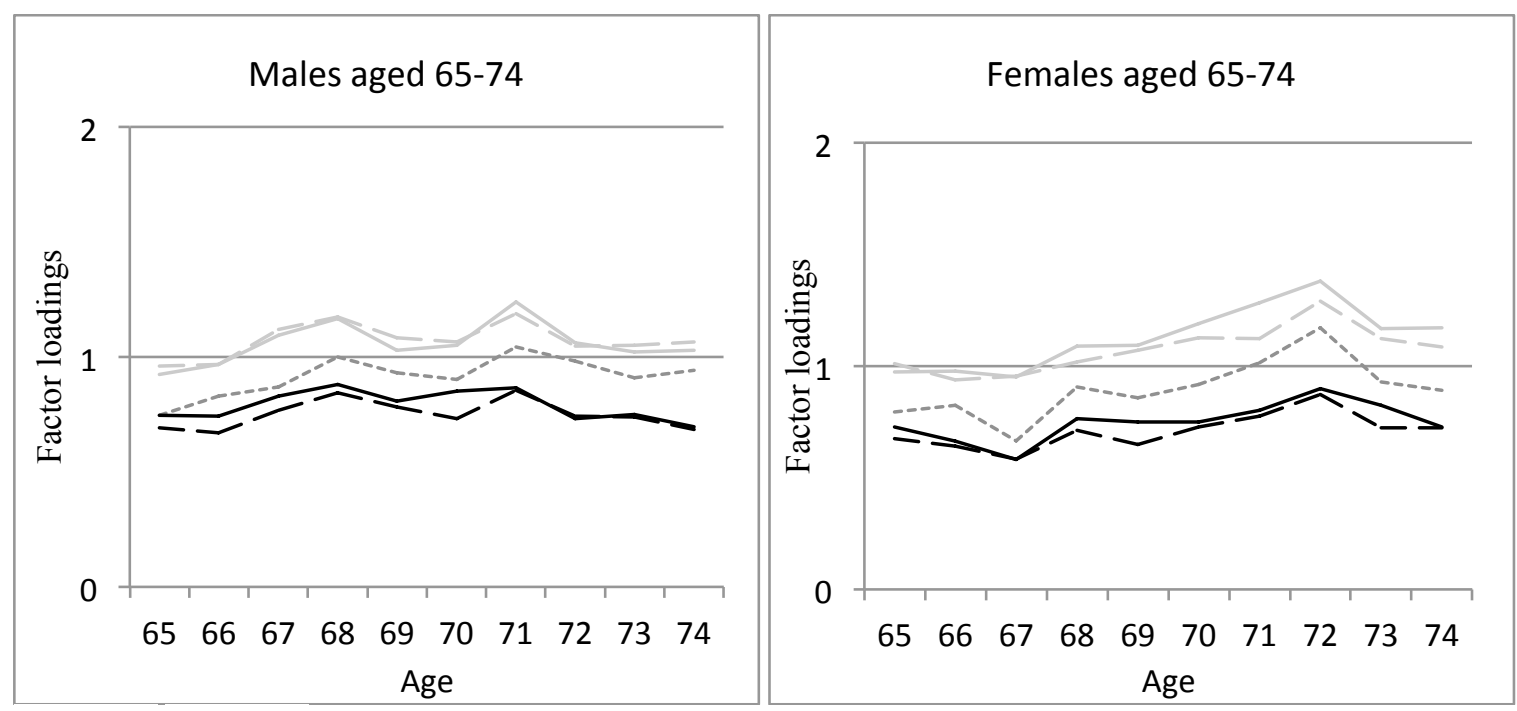

Walk down the road

Get up and down stairs

Bathe, shower or wash all over

$---\quad$ Get around the house

Get in and out of bed

Model 4 is our main model of interest, a full SEM combining a measurement model of the same specifications as model 3 (assuming metric and scalar invariance) with a growth model. We interpret the measurement model parameters below and in the following section we contrast the growth model parameters of model 4 with the alternative SEM which approximates a growth curve model fitted to an unweighted sum of scores on the ADL items (model 5). Model 5 is similar to the growth models fitted in most previous research, but with functional disability as a latent variable rather than a sum score.

An important consideration when evaluating differences in parameter estimates across subsamples (male vs. female) or model specifications (model 4 vs. model 5) is that these may be due in part to differences in the variance of the physical functioning factor. Suppose, for example, that we wish to compare the factor loading for a particular $A D L$ item for two groups. Even if the underlying relationship between the $A D L$ response and the factor is the same for each group, the estimated factor loading will be of smaller magnitude in the group with the largest factor variance. Standardised factor loadings and growth model coefficients can be computed to take account of such scaling effects (see Supplementary Data for details). We present unstandardised factor loadings and item intercepts for the measurement model component of the SEM in table 2, and unstandardised model estimates for all growth model parameters of models 4 and 5 in table 3. Between gender comparisons can be made as the factor variance is fairly similar across genders. However, because the factor variance changes according to whether or not the factor loadings in the measurement model are permitted to vary across $A D L$ items, we present a separate set of standardised estimates for the overall SES effects of Models 4 and 5 in Table 4. 
The measurement model component of the SEM

The factor loadings $\lambda_{r}$ and item intercepts $\alpha_{r}$ of the measurement part of the growth model are shown in table 2. The factor loadings are interpreted as the expected change in the observed $A D L$ item for a one-unit change in the factor, and represent the discriminatory power of the items in terms of the functional disability latent variable. The factor loading for the first item: 'cutting toenails' is constrained to one to fix the scale of the latent variable. 'Walking down the road' and 'managing steps' have the largest factor loadings indicating they are best at discriminating between individuals with different levels of functioning. 'Getting around the house' has the lowest factor loadings, followed by 'getting in and out of bed' then 'bathe, shower or wash all over' i.e. these are the least discriminatory items for changes in functional disability.

Table 2: Factor loadings $\lambda_{r}$ and item intercepts $\alpha_{r}$ for the measurement part of the SEM (Model 4). Standard errors are given in brackets.

\begin{tabular}{lll}
\hline & Males & Females \\
\hline Factor loadings $\left(\lambda_{r}\right):$ & 1 & 1 \\
$\quad$ Cut toenails & $1.013(0.023)$ & $1.058(0.024)$ \\
Walk down the road & $1.034(0.023)$ & $1.026(0.024)$ \\
Get up and down stairs or steps & $0.871(0.019)$ & $0.839(0.020)$ \\
Bath, shower or wash all over & $0.755(0.017)$ & $0.703(0.017)$ \\
Get in and out of bed & $0.716(0.016)$ & $0.664(0.016)$ \\
Get around the house & & 0 \\
Cut toenails & 0 & $-0.841(0.050)$ \\
Walk down the road & $-0.519(0.039)$ & $-0.611(0.049)$ \\
Get up and down stairs or steps & $-0.425(0.039)$ & $-0.692(0.040)$ \\
Bath, shower or wash all over & $-0.489(0.033)$ & $-0.594(0.033)$ \\
Get in and out of bed & $-0.444(0.028)$ & $-0.596(0.031)$ \\
Get around the house & $-0.442(0.027)$ & \\
\hline
\end{tabular}

The item intercepts represent the difficulty of the items. We constrain the first item 'cutting toenails' to zero, and this is the least difficult item because the estimated intercepts for the other items are all negative. For both genders 'walking down the road' is the most difficult item, followed by 'bathing, showering and washing'; the other categories ('managing stairs or steps', 'getting in and out of bed' and 'getting around the house') have roughly equal values for each gender. The intercepts are larger in magnitude for females, which is consistent with the literature on poorer female health.

\section{The growth model component of the SEM}

The parameter estimates for the growth model component of the full SEM (model 4) are shown in the left half of table 3 . The coefficients of the SES dummy variables are interpreted as contrasts with the reference group 'routine occupations' at the baseline age in the sample. Functional disability at baseline $\left(\beta_{0}\right)$ is greater for females. The intercept variances, $\operatorname{var}\left(u_{0 i}\right)$, are interpreted as the between-individual variance in the level of physical functioning at age $65(t=0)$ for each gender. We see a slightly larger baseline variance for females. 
Table 3: Growth model parameters and model fit statistics for Models 4 and 5, SEMs with unequal and equal factor loadings across ADL items.

\begin{tabular}{|c|c|c|c|c|c|c|c|c|}
\hline & \multicolumn{4}{|c|}{$\begin{array}{l}\text { Model 4: Growth model from SEM } \\
\text { with unequal factor loadings } \\
\text { for } A D L \text { items }\end{array}$} & \multicolumn{4}{|c|}{$\begin{array}{l}\text { Model 5: Growth model from SEM } \\
\text { with equal factor loadings } \\
\text { for ADL items }\end{array}$} \\
\hline & \multicolumn{2}{|c|}{ Male } & \multicolumn{2}{|c|}{ Female } & \multicolumn{2}{|c|}{ Male } & \multicolumn{2}{|c|}{ Female } \\
\hline \multicolumn{9}{|l|}{ Parameter estimates } \\
\hline Intercept growth factor mean $\left(\beta_{0}\right)$ & $1.098 * * *$ & $(0.067)$ & $1.443^{* * *}$ & $(0.065)$ & $1.102 * * *$ & $(0.058)$ & $1.478 * * *$ & $(0.055)$ \\
\hline Slope growth factor mean $\left(\beta_{1}\right)$ & $0.060 * * *$ & $(0.023)$ & $0.053^{*} *$ & $(0.021)$ & $0.048^{* *}$ & $(0.019)$ & $0.034^{*}$ & $(0.018)$ \\
\hline Quadratic growth factor mean $\left(\beta_{2}\right)$ & -0.001 & $(0.002)$ & 0.002 & $(0.002)$ & -0.001 & $(0.002)$ & 0.002 & $(0.002)$ \\
\hline \multicolumn{9}{|l|}{ Effects of NS-SEC on intercept $\left(\gamma_{0 m}\right)$ : } \\
\hline \multicolumn{9}{|l|}{ Routine occupations (reference) } \\
\hline Semi-routine occupations & 0.028 & $(0.096)$ & -0.023 & $(0.082)$ & 0.027 & $(0.079)$ & -0.017 & $(0.065)$ \\
\hline Lower supervisory and technical occupations & -0.047 & $(0.097)$ & $0.205^{*}$ & $(0.120)$ & -0.046 & $(0.079)$ & $0.157^{*}$ & $(0.095)$ \\
\hline Small employers and own account workers & -0.041 & $(0.091)$ & -0.127 & $(0.125)$ & -0.031 & $(0.074)$ & -0.093 & $(0.098)$ \\
\hline Intermediate occupations & $-0.386 * * *$ & $(0.136)$ & $-0.168^{*}$ & $(0.086)$ & $-0.305^{* * *}$ & $(0.111)$ & $-0.121 *$ & $(0.068)$ \\
\hline Lower managerial and professional occupations & $-0.185^{* *}$ & $(0.088)$ & $-0.160 *$ & $(0.084)$ & $-0.133^{*}$ & $(0.071)$ & $-0.125^{*}$ & $(0.066)$ \\
\hline Higher managerial and professional occupations & $-0.247 * *$ & $(0.103)$ & $-0.536 * *$ & $(0.209)$ & $-0.188 * *$ & $(0.084)$ & $-0.380 * *$ & $(0.163)$ \\
\hline \multicolumn{9}{|l|}{ Effects of NS-SEC on coefficient of $t\left(\gamma_{1 m}\right)$ : } \\
\hline \multicolumn{9}{|l|}{ Routine occupations (reference) } \\
\hline Semi-routine occupations & -0.030 & $(0.035)$ & -0.002 & $(0.030)$ & -0.027 & $(0.029)$ & -0.001 & $(0.025)$ \\
\hline Lower supervisory and technical occupations & 0.009 & $(0.036)$ & 0.003 & $(0.043)$ & 0.012 & $(0.030)$ & 0.002 & $(0.035)$ \\
\hline Small employers and own account workers & $-0.060^{*}$ & $(0.034)$ & -0.031 & $(0.046)$ & $-0.052^{*}$ & $(0.029)$ & -0.023 & $(0.038)$ \\
\hline Intermediate occupations & -0.034 & $(0.048)$ & -0.047 & $(0.031)$ & -0.024 & $(0.041)$ & -0.036 & $(0.026)$ \\
\hline Lower managerial and professional occupations & $-0.063 * *$ & $(0.032)$ & -0.021 & $(0.031)$ & $-0.051^{*}$ & $(0.027)$ & -0.011 & $(0.025)$ \\
\hline Higher managerial and professional occupations & $-0.107 * * *$ & $(0.038)$ & -0.031 & $(0.075)$ & $-0.087^{* * *}$ & $(0.032)$ & -0.026 & $(0.062)$ \\
\hline \multicolumn{9}{|l|}{ Effects of NS-SEC on coefficient of $t^{2}\left(\gamma_{2 m}\right)$ : } \\
\hline \multicolumn{9}{|l|}{ Routine occupations (reference) } \\
\hline Semi-routine occupations & 0.001 & $(0.004)$ & -0.001 & $(0.003)$ & 0.002 & $(0.003)$ & -0.001 & $(0.003)$ \\
\hline Lower supervisory and technical occupations & -0.002 & $(0.004)$ & -0.006 & $(0.004)$ & -0.002 & $(0.003)$ & -0.005 & $(0.004)$ \\
\hline Small employers and own account workers & 0.005 & $(0.004)$ & 0.001 & $(0.005)$ & $0.005^{*}$ & $(0.003)$ & 0.001 & $(0.004)$ \\
\hline Intermediate occupations & 0.005 & $(0.005)$ & 0.004 & $(0.003)$ & 0.004 & $(0.004)$ & 0.003 & $(0.003)$ \\
\hline Lower managerial and professional occupations & $0.005^{*}$ & $(0.003)$ & -0.001 & $(0.003)$ & 0.004 & $(0.003)$ & -0.001 & $(0.003)$ \\
\hline Higher managerial and professional occupations & $0.009 * *$ & $(0.004)$ & 0.005 & $(0.008)$ & $0.007^{*}$ & $(0.004)$ & 0.004 & $(0.007)$ \\
\hline Intercept growth factor variance, $\operatorname{var}\left(u_{0 i}\right)$ & $0.586 * * *$ & $(0.043)$ & $0.667 * * *$ & $(0.047)$ & $0.358 * * *$ & $(0.023)$ & $0.367 * * *$ & $(0.022)$ \\
\hline Slope growth factor variance, $\operatorname{var}\left(u_{1 i}\right)$ & $0.006 * * *$ & $(0.001)$ & $0.009 * * *$ & $(0.001)$ & $0.004 * * *$ & $(0.001)$ & $0.005^{* * *}$ & $(0.001)$ \\
\hline Covariance between factor mean and slope, $\operatorname{cov}\left(u_{0 i}, u_{1 i}\right)$ & $-0.010 * *$ & $(0.005)$ & -0.007 & $(0.005)$ & $-0.007^{* *}$ & $(0.003)$ & $-0.006 * *$ & $(0.003)$ \\
\hline Residual variance for the factor, $\operatorname{var}\left(e_{i}\right)$ & $0.217^{* * *}$ & $(0.010)$ & $0.213^{* * *}$ & $(0.010)$ & $0.164 * * *$ & $(0.004)$ & $0.156^{* * *}$ & $(0.004)$ \\
\hline \multicolumn{9}{|l|}{ Model fit } \\
\hline Chi-square test statistic & \multicolumn{3}{|c|}{$5,531(1,885 d f)$} & $3(1,885 \mathrm{df})$ & \multicolumn{2}{|c|}{$6,489(1,890 \mathrm{df})$} & \multicolumn{2}{|c|}{$6,645(1,890 \mathrm{df})$} \\
\hline TLI & \multicolumn{2}{|c|}{0.898} & \multicolumn{2}{|c|}{0.906} & \multicolumn{2}{|c|}{0.871} & \multicolumn{2}{|c|}{0.870} \\
\hline RMSEA & \multicolumn{2}{|c|}{0.034} & \multicolumn{2}{|c|}{0.030} & \multicolumn{2}{|c|}{0.038} & \multicolumn{2}{|c|}{0.036} \\
\hline SRMSR & 0.0 & 75 & & 072 & & 125 & & 131 \\
\hline$n$ & 1,7 & 12 & & 959 & 1,7 & 712 & & 959 \\
\hline
\end{tabular}


Table 4: Comparison of standardised SES effects for growth model component of Models 4 and 5, SEMs with unequal and equal factor loadings across ADL items.

\begin{tabular}{|c|c|c|c|c|c|c|c|c|}
\hline \multirow[b]{2}{*}{ Age } & \multicolumn{4}{|c|}{$\begin{array}{c}\text { Model 4: Growth model from SEM } \\
\text { with unequal factor loadings } \\
\text { for ADL items }\end{array}$} & \multicolumn{4}{|c|}{$\begin{array}{c}\text { Model 5: Growth model from SEM } \\
\text { with equal factor loadings } \\
\text { for ADL items }\end{array}$} \\
\hline & 65 & 68 & 71 & 74 & 65 & 68 & 71 & 74 \\
\hline \multicolumn{9}{|l|}{ Males: } \\
\hline Routine occupations & 0 & 0 & 0 & 0 & 0 & 0 & 0 & 0 \\
\hline Semi-routine occupations & 0.031 & -0.059 & -0.122 & -0.153 & 0.037 & -0.050 & -0.083 & -0.064 \\
\hline Lower supervisory and technical occupations & -0.052 & -0.043 & -0.069 & -0.122 & -0.064 & -0.039 & -0.060 & -0.118 \\
\hline Small employers and own account workers & -0.046 & -0.197 & -0.233 & -0.167 & -0.043 & -0.198 & -0.214 & -0.111 \\
\hline Intermediate occupations & -0.431 & -0.496 & -0.432 & -0.273 & -0.422 & -0.475 & -0.400 & -0.232 \\
\hline Lower managerial and professional occupations & -0.206 & -0.369 & -0.404 & -0.330 & -0.184 & -0.348 & -0.387 & -0.316 \\
\hline Higher managerial and professional occupations & -0.276 & -0.546 & -0.596 & -0.457 & -0.260 & -0.537 & -0.600 & -0.476 \\
\hline \multicolumn{9}{|l|}{ Females: } \\
\hline Routine occupations & 0 & 0 & 0 & 0 & 0 & 0 & 0 & 0 \\
\hline Semi-routine occupations & -0.025 & -0.040 & -0.067 & -0.100 & -0.024 & -0.040 & -0.074 & -0.118 \\
\hline Intermediate occupations & -0.179 & -0.285 & -0.289 & -0.219 & -0.167 & -0.277 & -0.288 & -0.223 \\
\hline Lower managerial and professional occupations & -0.171 & -0.242 & -0.304 & -0.353 & -0.173 & -0.229 & -0.286 & -0.337 \\
\hline Higher managerial and professional occupations & -0.571 & -0.609 & -0.512 & -0.337 & -0.525 & -0.579 & -0.493 & -0.320 \\
\hline
\end{tabular}

Predicted trajectories for each gender are presented in figure 3 with separate curves for each SES group. These trajectories are calculated using the SES coefficients for functional disability for someone at the mean of the distribution, in other words the individual random effects are set at their means of zero. For all SES groups we estimate a positive linear growth $\left(\beta_{1}\right)$ in functional disability, and the quadratic growth factor mean $\left(\beta_{2}\right)$ shows a slight acceleration in growth for females but not for males. The random effect variance associated with the linear age effect, $\operatorname{var}\left(u_{1 i}\right)$, is similar for men and women, though slightly smaller for males. There is a negative covariance (though statistically insignificant for females) between the individual intercepts and slopes suggesting that higher functional disability at baseline is associated with slower increase in functional disability over time. Note that the variance of the random effect for $t^{2}$ $\left(u_{2 i}\right)$ and its covariances with the other random effects were found to be negligible, and were therefore omitted from the structural model. 
Figure 3: Functional disability trajectories by socioeconomic status (Model 4)

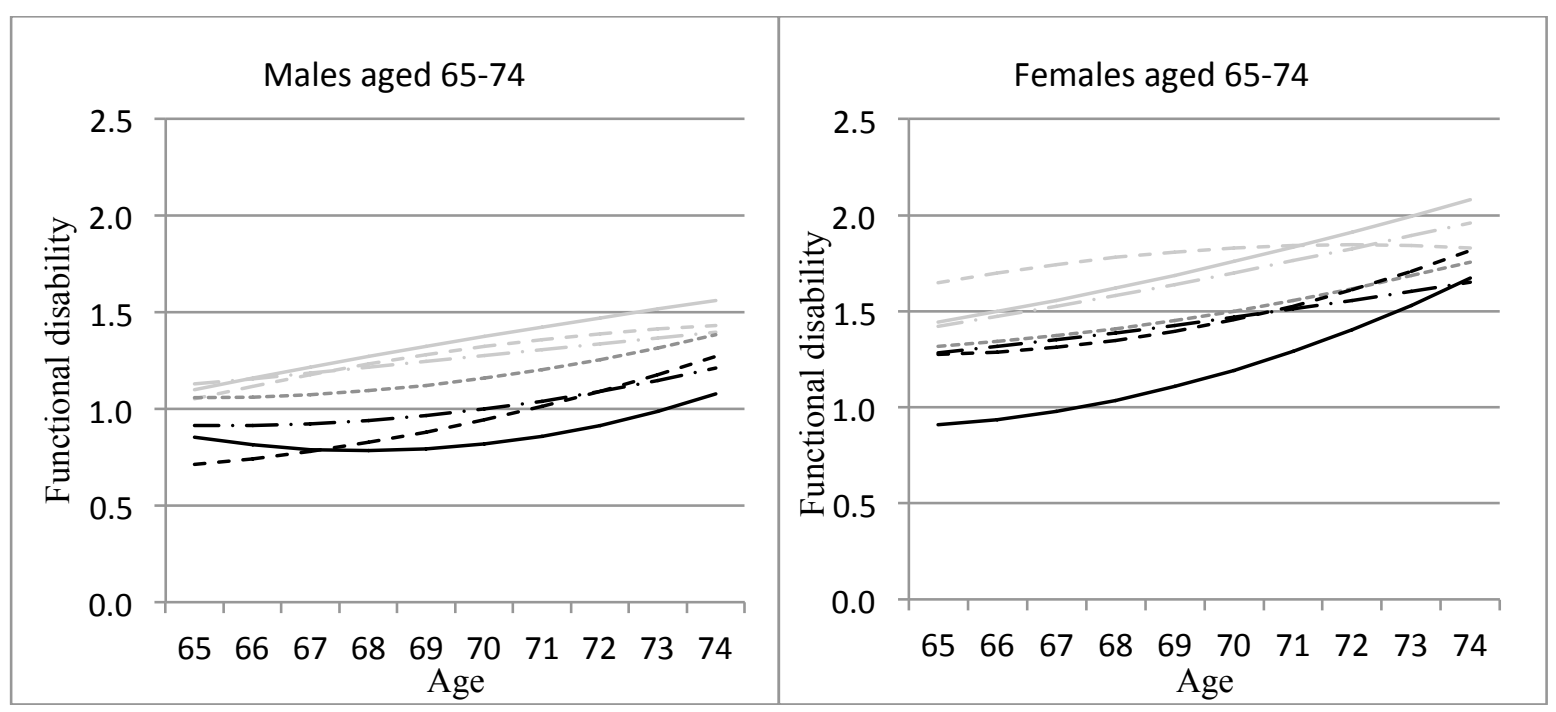

\footnotetext{
- Routine occupations

- - Semi-routine occupations

Lower supervisory and technical occupations

Small employers and own account workers

Intermediate occupations

- L L Lower managerial and professional occupations

Higher managerial and professional occupations
}

SES is allowed to affect both the intercept and slope of functional disability. For each gender we find small but significant effects of SES on the intercept $\left(\gamma_{0 m}\right)$ compared with the reference category 'routine occupations', though the lower status occupations ('small employers and own account workers', 'lower supervisory and technical occupations', and 'semi-routine occupations') are not statistically significantly different. In terms of the social gradient in the change in functional disability $\left(\gamma_{1 m}\right.$ and $\left.\gamma_{2 m}\right)$ males show a slight widening of the social gradient in functional disability with age, while females show a slight convergence with age (though from a more divergent baseline), though these relationships are only statistically significant for males and only for the less routine occupations.

In the right-hand side of table 4 we show estimates from a comparison model (model 5) which proxies a growth model fitted to an unweighted sum of ADL scores. Table 5 shows standardised SES effects for models 4 and 5 for the male and female subsamples, calculated for selected ages three years apart. We would not expect the SES effects to be dramatically different given the factor loadings from the measurement part of model 4 shown in table 2 are relatively close to one another. This comparison shows the SES effects would be slightly underestimated when no measurement models is used, with the most noticeable differences for males at the older ages (71 and 74).

\section{Discussion}

The general health of the elderly population is typically measured using questions relating to functional ability across a range of dimensions. When using these measures to model trajectories 
of functional disability as people age, researchers typically use simple methods to combine these indicators, such as the total score. We argue that these approaches are limited since they do not capture the difference in discriminatory power of these different items. We propose supplementing the growth model of functional disability with a measurement model to better capture the underlying latent variable functional disability that we wish to use as the outcome in the growth model.

Another advantage of specifying a measurement model is that it makes explicit and allows testing of the assumption of temporal measurement invariance. We estimated a sequence of three increasingly restricted models in order to test for measurement invariance for the gender subsamples. Vandenberg and Lance (2000) argue that assessing model fit using only a $\chi^{2}$ test is limited because it is sensitive to sample size and differences in the covariance structure, and suggest using a suite of fit indices including TLI, RMSEA and SRMR to evaluate the degree of temporal measurement invariance. By recognising the strengths and weaknesses of each of these indices we are able to build a more robust assessment of the temporal measurement invariance assumption.

We estimate SEM of the growth in latent functional disability separately for each gender. Overall we see increasing functional disability, with accelerating growth for males but not for females. For both genders we find evidence of a social gradient in the baseline levels of functional disability between the most routine occupational class (the reference category) and the least routine social classes. The social gradient in the rate of change of functional disability was less clear. Our model predicts that the functional disability of an individual from the lowest SES group at baseline (aged 65) is equivalent to that of an individual from the highest SES group who was ten years older for males and seven years older for females.
Future research which estimates trajectories of functional disability for the elderly could benefit from adopting our approach of using a SEM to incorporate a measurement model which treats functional disability as a latent variable. This includes work using richer datasets, which would allow a wider set of items to measure functional disability and a wider set of controls. For example the English Longitudinal Study of Ageing includes measures of $\mathrm{iADL}$ and mobility to supplement the $A D L$, and has better measures of SES to improve identification of the social gradient in functional disability trajectories (which could be used in a second measurement model for a latent SES measure). To date, these data have only been used to model functional disability cross-sectionally (Gjonça, Tabassum, \& Breeze, 2009). Time-varying measures of social status would allow us to explore the relationship between change in SES and change in functional disability, and determine whether the changes in SES effects with age are real or simply a function of increasing time since the measure was taken. We know that there may be reverse causality in this relationship as health status could also impact on social status (Steele, French, \& Bartley, 2013). Longitudinal data on both health and SES would allow us to identify the direction of these effects. Residential status is another time-varying characteristic of policy relevance (because of the cost of residential care), which may be included as a determinant of functional disability trajectories. Such a model could be extended to identify the effect of residential status on individuals where care needs (including moves into residential care) are not met (Scott, Evandrou, Falkingham, \& Rake, 2001). Finally, studies that incorporate this approach over shorter-term periods would be able to capture aspects of recovery as well as the longer term increase in disability found in this study. Importantly, a shorter time span would also make it easier to satisfy the temporal measurement invariance assumption.

\section{Acknowledgements}

This work was supported by the UK Economic and Social Research Council (grant number RES-576-25-0032). 


\section{References}

Bentler, P. M. (1995). EQS structural equations program manual (Vol. 6).

Burchardt, T. (2000). The dynamics of being disabled. Journal of Social Policy, 29(04), 645-668. http://dx.doi.org/10.1017/S0047279400006097

Case, A., \& Paxson, C. (2005). Sex differences in morbidity and mortality. Demography, 42(2), 189-214. http://dx.doi.org/10.1353/dem.2005.0011

Chan, D. (1998). The conceptualization and analysis of change over time: an integrative approach incorporating Longitudinal Mean and Covariance Structures Analysis (LMACS) and Multiple Indicator Latent Growth Modeling (MLGM). Organizational Research Methods, 1(4), 421-483. http://dx.doi.org/10.1177/109442819814004

Cheung, G. W., \& Rensvold, R. B. (1999). What constitutes significant differences in evaluating measurement invariance? Paper presented at the 1999 conference of the Academy of Management, Chicago.

Curran, P. J. (2003). Have multilevel models been structural equation models all along? Multivariate Behavioral Research, 38(4), 529-569. http://dx.doi.org/10.1207/s15327906mbr3804_5

Feng, Q., Zhen, Z., Gu, D., Wu, B., Duncan, P. W., \& Purser, J. L. (2013). Trends in ADL and IADL disability in community-dwelling older adults in Shanghai, China, 1998-2008. The Journals of Gerontology Series B: Psychological Sciences and Social Sciences, 68(3), 476-485. http://dx.doi.org/10.1093/geronb/gbt012

Fries, J. F. (1980). Aging, natural death, and the compression of morbidity. New England Journal of Medicine, 303(3), 130-135. http://dx.doi.org/10.1056/NEJM198007173030304

Gjonça, E., Tabassum, F., \& Breeze, E. (2009). Socioeconomic differences in physical disability at older age. Journal of Epidemiology and Community Health, 63(11), 928-935. http://dx.doi.org/10.1136/jech.2008.082776

Goldstein, H., \& Woodhouse, G. (2001). Modelling repeated measures. In A. H. Leyland \& H. Goldstein (Eds.), Multilevel modelling of health statistics (pp. 13-26). Chichester: Wiley.

Grundy, E., \& Glaser, K. (2000). Socio-demographic differences in the onset and progression of disability in early old age: a longitudinal study. Age and Ageing, 29(2), 149-157. http://dx.doi.org/10.1093/ageing/29.2.149

Haas, S. (2008). Trajectories of functional health: The 'long arm' of childhood health and socioeconomic factors. Social Science \& Medicine, 66(4), 849-861.

http://dx.doi.org/10.1016/j.socscimed.2007.11.004

Hancock, G. R., Kuo, W.-L., \& Lawrence, F. R. (2001). An illustration of second-order latent growth models. Structural Equation Modeling: a Multidisciplinary Journal, 8(3), 470-489. http://dx.doi.org/10.1207/S15328007SEM0803_7

Holstein, B. E., Avlund, K., Due, P., Martinussen, T., \& Keiding, N. (2006). The measurement of change in functional ability: dealing with attrition and the floor/ceiling effect. Archives of Gerontology and Geriatrics, 43(3), 337-350. http://dx.doi.org/10.1016/j.archger.2005.12.004

House, J. S., Kessler, R. C., \& Herzog, A. R. (1990). Age, socioeconomic status, and health. The Milbank Quarterly, 68(3), 383-411. http://dx.doi.org/10.2307/3350111

Institute for Social \& Economic Research (2010). British Household Panel Survey: waves 1-18, 1991-2009 [computer file] 7th Edition. Retrieved from: http://discover.ukdataservice.ac.uk/catalogue?sn=5151

Hu, L. t., \& Bentler, P. M. (1999). Cutoff criteria for fit indexes in covariance structure analysis: conventional criteria versus new alternatives. Structural Equation Modeling: a Multidisciplinary Journal, 6(1), 1-55. http://dx.doi.org/10.1080/10705519909540118

Katz, S., Ford, A., Moskowitz, R., Jackson, B., \& Jaffe, M. (1963). Studies of illness in the aged. The index of ADL: a standardized measure of biological and psychosocial function. Journal of the American Medical Association, 185, 914 - 919. http://dx.doi.org/10.1001/jama.1963.03060120024016

Kline, R. B. (2005). Principles and practice of structural equation modelling $\left(2^{\text {nd }}\right.$. ed.). New York: The Guilford Press. 
LaPlante, M. P. (2010). The classic measure of disability in activities of daily living Is biased by age but an expanded IADL/ADL measure is not. The Journals of Gerontology Series B: Psychological Sciences and Social Sciences, 65B(6), 720-732. http://dx.doi.org/10.1093/geronb/gbp129

Lawton, M. P., \& Brody, E. M. (1969). Assessment of older people: Self-maintaining and instrumental activities of daily living. Gerontologist, 9, 179-186. http://dx.doi.org/10.1093/geront/9.3_Part_1.179

Li, L. W. (2005). Trajectories of ADL disability among community-dwelling frail older persons. Research on Aging, 27(1), 56-79. http://dx.doi.org/10.1177/0164027504271348

Little, R. J. A., \& Rubin, D. B. (2002). Statistical analysis with missing data. New York: Wiley. http://dx.doi.org/10.1002/9781119013563

Mendes de Leon, C. F., Guralnik, J. M., \& Bandeen-Roche, K. (2002). Short-term change in physical function and disability: the Women's Health and Aging Study. The Journals of Gerontology Series B: Psychological Sciences and Social Sciences, 57(6), S355-S365. http://dx.doi.org/10.1093/geronb/57.6.S355

Muthén, L. K., \& Muthén, B. O. (1998-2012). Mplus user's guide. Seventh Edition. Los Angeles, CA: Muthén \& Muthén.

Nathanson, C. A. (1975). Illness and the feminine role: a theoretical review. Social Science \& Medicine (1967), 9(2), 57-62.

Nilsson, C. J., Avlund, K., \& Lund, R. (2010). Social inequality in onset of mobility disability among older Danes: the mediation effect of social relations. Journal of Aging and Health, 22(4), 522-541. http://dx.doi.org/10.1177/0898264309359684

Office for National Statistics. (2010). The National Statistics socio-economic classification: (Rebased on the SOC2010) User Manual (Vol. 3). Southampton: Palgrave Macmillan.

Park, N. S., Klemmack, D. L., Roff, L. L., Parker, M. W., Koenig, H. G., Sawyer, P., \& Allman, R. M. (2008). Religiousness and longitudinal trajectories in elders' functional status. Research on Aging, 30(3), 279298. http://dx.doi.org/10.1177/0164027507313001

Payette, H., Gueye, N. D. R., Gaudreau, P., Morais, J. A., Shatenstein, B., \& Gray-Donald, K. (2011). Trajectories of physical function decline and psychological functioning: the Québec Longitudinal Study on Nutrition and Successful Aging (NuAge). The Journals of Gerontology Series B: Psychological Sciences and Social Sciences, 66B(suppl 1), i82-i90. http://dx.doi.org/10.1093/geronb/gbq085

Sacker, A., Clarke, P., Wiggins, R. D., \& Bartley, M. (2005). Social dynamics of health inequalities: a growth curve analysis of aging and self assessed health in the British Household Panel Survey 1991-2001. Journal of Epidemiology and Community Health, 59(6), 495-501. http://dx.doi.org/10.1136/jech.2004.026278

Sacker, A., Worts, D., \& McDonough, P. (2011). Social influences on trajectories of self-rated health: evidence from Britain, Germany, Denmark and the USA. Journal of Epidemiology and Community Health, 65(2), 130-136. http://dx.doi.org/10.1136/jech.2009.091199

Scott, A., Evandrou, M., Falkingham, J., \& Rake, K. (2001). Moves into residential care amongst older people in Britain. Paper Presented to the 2001 British Household Panel Survey Research Conference; 5-7 July, 2001, Colchester, UK, 2012 (November). https://www.iser.essex.ac.uk/files/conferences/bhps/2001/docs/pdf/papers/scott.pdf

Steele, F. (2008). Multilevel models for longitudinal data. Journal of the Royal Statistical Society: Series A (Statistics in Society), 171(1), 5-19.

doi: 10.1111/j.1467-985X.2007.00509.x

Steele, F., French, R., \& Bartley, M. (2013). Adjusting for selection bias in longitudinal analyses using simultaneous equations modeling: the relationship between employment transitions and mental health. Epidemiology, 24(5), 703-711. http://dx.doi.org/10.1097/EDE.0b013e31829d2479

Steiger, J. (1990). Structural model evaluation and modification: an interval estimation approach. Multivariate Behavioral Research, 25(2), 173-180. http://dx.doi.org/10.1207/s15327906mbr2502_4

Stuck, A. E., Walthert, J. M., Nikolaus, T., Büla, C. J., Hohmann, C., \& Beck, J. C. (1999). Risk factors for functional status decline in community-living elderly people: a systematic literature review. Social Science \& Medicine, 48(4), 445-469. http://dx.doi.org/10.1016/S0277-9536(98)00370-0 
Tucker, L. R., \& Lewis, C. (1973). A reliability coefficient for maximum likelihood factor analysis. Psychometrika, 38(1), 1-10. http://dx.doi.org/10.1007/BF02291170

Vandenberg, R. J., \& Lance, C. E. (2000). A review and synthesis of the measurement invariance literature: suggestions, practices, and recommendations for organizational research. Organizational Research Methods, 3(1), 4-70. http://dx.doi.org/10.1177/109442810031002

Verbrugge, L. M. (1984). Longer life but worsening health? Trends in health and mortality of middle-aged and older persons. The Milbank Memorial Fund Quarterly. Health and Society, 62(3), 475-519. http://dx.doi.org/10.2307/3349861

Verbrugge, L. M. (1989). The twain meet: empirical explanations of sex differences in health and mortality. Journal of Health and Social Behavior, 30(3), 282-304. http://dx.doi.org/10.2307/2136961

Ware, J. E. J., \& Sherbourne, C. D. (1992). The MOS 36-Item Short-Form health survey (SF-36). I. Conceptual framework and item selection. Medical Care, 30(6), 473-483. http://dx.doi.org/10.1097/00005650-199206000-00002

Wu, A., Liu, Y., Gadermann, A., \& Zumbo, B. (2010). Multiple-indicator multilevel growth model: a solution to multiple methodological challenges in longitudinal studies. Social Indicators Research, 97(2), 19. http://dx.doi.org/10.1007/s11205-009-9496-8

Zaninotto, P., Falaschetti, E., \& Sacker, A. (2009). Age trajectories of quality of life among older adults: results from the English Longitudinal Study of Ageing. Quality of Life Research, 18(10), 1301-1309. http://dx.doi.org/10.1007/s11136-009-9543-6 